\title{
Inhibition of p38 MAPK regulates epileptic severity by decreasing expression levels of A1R and ENT1
}

\author{
XUEJIAO ZHOU $^{1 *}$, QIAN CHEN ${ }^{1}{ }^{*}$, HAO HUANG $^{1}$, JUN ZHANG $^{1}$, JING WANG $^{2}$, YA CHEN $^{1}$, \\ YAN PENG ${ }^{1}$, HAIQING ZHANG ${ }^{1}$, JUNWEI ZENG ${ }^{3}$, ZHANHUI FENG ${ }^{4}$ and ZUCAI XU ${ }^{1}$ \\ Departments of ${ }^{1}$ Neurology and ${ }^{2}$ Prevention and Health Care, Affiliated Hospital of Zunyi Medical University; \\ ${ }^{3}$ Department of Physiology, Zunyi Medical University, Zunyi, Guizhou 563003; ${ }^{4}$ Department of Neurology, \\ Affiliated Hospital of Guizhou Medical University, Guiyang, Guizhou 550004, P.R. China
}

Received January 15, 2020; Accepted September 8, 2020

DOI: $10.3892 / \mathrm{mmr} .2020 .11614$

\begin{abstract}
Epilepsy is a chronic nervous system disease. Excessive increase of the excitatory neurotransmitter glutamate in the body results in an imbalance of neurotransmitters and excessive excitation of neurons, leading to epileptic seizures. Long-term recurrent seizures lead to behavior and cognitive changes, and even increase the risk of death by 2 - to 3 -fold relative to the general population. Adenosine A1 receptor (A1R), a member of the adenosine system, has notable anticonvulsant effects, and adenosine levels are controlled by the type 1 equilibrative nucleoside transporter (ENT1); in addition the p38 MAPK signaling pathway is involved in the regulation of ENT1, although the effect of its inhibitors on the expression levels of A1R and ENT1 is unclear. Therefore, in the present study, SB203580 was used to inhibit the p38 MAPK signaling pathway in rats, and the expression levels of A1R and ENT1 in the brain tissue of rats with acute $\mathrm{LiCl}$-pilocarpine-induced status epilepticus was detected. SB203580 decreased pathological damage of hippocampal neurons, prolonged seizure latency, reduced the frequency of seizures, and decreased levels of A1R and ENT1 protein in rats.
\end{abstract}

\section{Introduction}

Epilepsy is one of the most common and serious chronic diseases of the nervous system. According to the World Health Organization, 70 million individuals currently suffer from epilepsy worldwide (1). The imbalance of excitatory and inhibitory neurotransmission is a common mechanism

Correspondence to: Professor Zucai Xu, Department of Neurology, Affiliated Hospital of Zunyi Medical University, 149 Dalian Road, Zunyi, Guizhou 563003, P.R. China

E-mail: doctorxzc@126.com

${ }^{*}$ Contributed equally

Key words: status epilepticus, adenosine A1 receptor, equilibrative nucleoside transporter, p38 MAPK, SB203580 of epilepsy. Status epilepticus (SE) is a state in which failure of the epileptic termination mechanism or an abnormality of the epileptic initiation mechanism leads to long-term epileptic seizures and requires emergency administration of antiepileptic drugs (2). Failure to terminate SE over time results in neuronal damage and death, and neural network changes (3). Pilocarpine injection can result in SE, which triggers a series of molecular and cellular events that produce neuronal cell death and can culminate in the development of epilepsy $(4,5)$. In a previous study, the p38 signal transduction pathway was activated in kainate-induced status epilepticus and was involved in neuronal damage, moss sprouting, glial cell proliferation and various other changes in the CA1 and CA3 areas of the hippocampus following SE (6). Activation of the p38 pathway can cause damage to hippocampal neurons and increases susceptibility to seizures (7). In addition, the mechanism of p38 signaling pathway may also be associated with its involvement in gliosis following seizures (8). Although there are a number of antiepileptic drugs that can effectively control epileptic seizures, certain cases of seizures cannot be controlled over time, resulting in morbidity and mortality rates $\sim 20 \%$ (9). Therefore, exploring the pathophysiological mechanism of SE and providing novel therapeutic targets for antiepileptic drugs are urgently required.

Excessive activity of the excitatory neurotransmitter glutamate results in an imbalance of excitability and inhibition in vivo, causing excessive neuronal excitability, leading to a seizure (10). The anticonvulsant effect of the adenosine system has been confirmed in a number of epilepsy models, suggesting that the adenosine system serves an important role in inhibiting neuronal excitation $(11,12)$. Adenosine has been demonstrated to be an endogenous anticonvulsant and neuroprotective agent in the brain, and its extracellular levels are largely dependent on ectonucleotidase-mediated conversion of 'activated' synapse-released ATP and the secretion of astrocytes (13). However, two-way regulation of adenosine levels also depends on type 1 equilibrative nucleoside transporter (ENT1) (13). In addition, the anticonvulsant and neuroprotective effects of adenosine are largely mediated by adenosine $\mathrm{A} 1$ receptor (A1R) on the presynaptic membrane (11). The activity-dependent release of adenosine activates A1R to block the release of excitatory transmitters, such as glutamate (14). 
Therefore, A1R is an effective molecular and therapeutic target in the course of epileptic seizures (12). Moreover, activated A1R provides a potential endogenous mechanism to inhibit neuronal excitatory activity and seizure dispersion (15).

MAPKs mediate intracellular signaling cascades in response to a variety of stimuli by regulating intracellular gene expression levels, cell division, differentiation, repair and apoptosis (16). MAPKs are activated by extracellular stressors, such as UV light, osmotic pressure, radiation, inflammatory cytokines, growth factors and shock, and subsequently induce a cascade of three kinases to transduce signals (17). Upon stimulation, MAPK kinase kinase is activated by phosphorylation and then phosphorylates and activates MAPK kinase, which in turn phosphorylates and activates MAPK (17). p38 is a MAPK primarily activated by inflammatory factors, stressful stimuli, such as UV light exposure, hypertonic conditions and heat shock, and glutamate (18). Okamoto et al (19) performed a whole transcriptome analysis of the hippocampi of epileptic rats and observed that p38 MAPK was overexpressed. Our previous study (20) confirmed that the specific p38 MAPK inhibitor SB203580 alleviated epileptic seizures in SE rats. Nitrobenzothioinosine (NBTI), a selective inhibitor of ENT1 (21), blocks the transport of adenosine into cells and increases extracellular adenosine levels, thus exhibiting a protective effect against epileptic seizures (22). A number of studies have revealed that A1R, a member of the adenosine system, has significant anticonvulsant effects $(13,23)$. p38 MAPK affects the levels of ENT1 (24). However, whether the p38 MAPK-specific inhibitor SB203580 regulates the expression levels of A1R and ENT1, and exerts a protective effect during seizures remains unclear.

In the present study, SB203580 was used to inhibit the p38 MAPK signaling pathway in rats. It was observed that SB203580 could reduce the pathomorphological damage of hippocampal neurons, prolong the latency of seizure and reduce the degree of seizure in rats, and reduce the expression of A1R and ENT1.

\section{Materials and methods}

Experimental animals. In total, 50 healthy adult male Sprague-Dawley (SD) (age, 42-49 days) were purchased from the Experimental Animal Center of Daping Hospital of the Third Military Medical University [license no. scxk (Chongqing, China) 2012-0005]. The rats were provided a standard diet and pure drinking water ad libitum in a room maintained at a constant temperature $\left(24 \pm 2^{\circ} \mathrm{C}\right.$, and $50-70 \%$ humidity. Animal health and behavior were monitored every day; all rats were healthy during the experimental period. The experiment lasted for 2 weeks. All efforts were made to minimize animal suffering. All procedures were approved by the Animal Care and Use Committee of Zunyi Medical University (Zunyi, China).

SD rats (weight, $\sim 180-220 \mathrm{~g}$ ) were randomly divided into the following 10 groups (5 rats per group): i) Control; ii) NBTI; iii) NBTI + SB203580; iv) SE, 0 h; v) SE, 1 day; vi) SE, 3 days; vii) SE, 7 days; viii) SE, 14 days; ix) SB203580; and x) DMSO. A rat model of acute LiCl-pilocarpine SE was established as described below. Then, randomly selected rats in the SE group received $127 \mathrm{mg} / \mathrm{kg} \mathrm{LiCl}$ by intraperitoneal (i.p.) injection, followed by $1 \mathrm{mg} / \mathrm{kg}$ atropine sulfate i.p. $18-20 \mathrm{~h}$ later, then $50 \mathrm{mg} / \mathrm{kg}$ pilocarpine i.p. after an additional $30 \mathrm{~min}$. A Racine score of IV-V were recorded (25) to statistical analysis of rat behavior.

For the control rats, instead of the $\mathrm{LiCl}$ and pilocarpine administered to the SE group, the same amount of saline was used instead. For rats in the SB20358 group, a solution of the p38 inhibitor SB203580 (15 mg/kg) and 2\% DMSO was injected i.p. $30 \mathrm{~min}$ prior to injection of pilocarpine (26-28). For rats in the NBTI group, an ENT1 inhibitor (15 mg/kg) (29) was injected i.p. 45 min prior to injection of pilocarpine and $2 \%$ DMSO. For rats in the NBTI + SB20358 group, the same protocol was used as aforementioned. For rats in the DMSO group, an equal volume of saline and $2 \%$ DMSO was used as a substitution for SB203580.

Euthanasia was accomplished humanely by a trained laboratory personnel via i.p. injection of saline-diluted Euthasol ${ }^{\circledR}$ $(150 \mathrm{mg} / \mathrm{kg})$ containing pentobarbital sodium $(390 \mathrm{mg} / \mathrm{ml})$ and phenytoin sodium $(50 \mathrm{mg} / \mathrm{ml})$, followed by cervical dislocation at the experimental timepoints ( $0 \mathrm{~h}, 1,3,7$ and 14 days). The death of rats was verified using the following criteria: Lack of pulse, breathing and corneal reflex, failure to respond to firm toe pinch, graying of mucus membranes and inability to auscultate respiratory or heart sounds. Following sacrifice of rats, the skull was dissected, brain tissue was removed and the bilateral hippocampus and temporal lobe neocortex were rapidly dissected on ice. Tissue samples were frozen in liquid nitrogen immediately and stored until western blot analysis. After administering complete anesthesia, the control group, SE group and SB203580 group were injected with saline $(\sim 150 \mathrm{ml})$ rapidly and continuously. Then, $4 \%$ paraformaldehyde $(\sim 150 \mathrm{ml})$ was injected at room temperature until the limbs, neck and tail became stiff. Then, rats were decapitated and the brain tissue was removed, incubated in $4 \%$ paraformaldehyde for $12-24 \mathrm{~h}\left(4^{\circ} \mathrm{C}\right)$ and sent to the Department of Pathology of Zunyi Medical College to be embedded in paraffin wax and stored at room temperature prior to immunohistochemistry.

Tissues for immunofluorescence and immunohistochemistry were prepared in the same manner. Following decapitation, the brain tissue was placed in successive 20 and $30 \%$ sucrose solutions for dehydration at $4^{\circ} \mathrm{C}$ for $24 \mathrm{~h}$ each time; after brain tissue sank, it was sent to the Zunyi Medical College Affiliated Hospital for the preparation of frozen microtome sections (thickness, $16 \mu \mathrm{m}$ ) and stored at $-20^{\circ} \mathrm{C}$.

Western blotting. RIPA buffer and protease inhibitors (Beyotime Institute of Biotechnology) were used to homogenize all samples. The protein concentration of the supernatant was measured with a BCA kit (Beyotime Institute of Biotechnology) according to the manufacturer's instructions. A total of $50 \mu \mathrm{g}$ protein from each sample was separated via SDS-PAGE (5\% separating, $10 \%$ stacking gel), and then proteins were transferred to PVDF membranes $(250 \mathrm{~mA}$ for $100 \mathrm{~min})$. After blocking for $1 \mathrm{~h}$ at room temperature in skimmed milk (5\%), PVDF membranes were incubated with primary antibodies [rabbit anti-A1R (1:1,000; cat. no. ab82477; Abcam) and anti-ENT1 (1:500; cat. no. ab223851; Abcam) and mouse anti- $\beta$-actin (1:5000; cat. no. 66009-1-Ig; ProteinTech Group) and anti- $\beta$-tubulin (1:5,000; cat. no. 10094-1-AP; ProteinTech Group)] overnight at $4^{\circ} \mathrm{C}$. The blots were washed 
Table I. SB203580 influences the latency of the first seizure and the number of epileptic seizures in rats.

\begin{tabular}{lcc}
\hline Group & Latency of seizures (min) & Number of epileptic seizures, $\mathrm{n}$ \\
\hline SE & $25.89 \pm 1.27$ & $7.40 \pm 1.07$ \\
SB203580 & $37.53 \pm 1.73^{\mathrm{a}}$ & $3.00 \pm 0.70^{\mathrm{a}}$ \\
DMSO & $25.65 \pm 1.56$ & $7.00 \pm 0.83$ \\
\hline
\end{tabular}

${ }^{\mathrm{a}} \mathrm{P}<0.05$ vs. SE. The number of epileptic seizures was observed continuously for $1 \mathrm{~h}$ and is presented as the mean $\pm \mathrm{SD}$. SE, status epilepticus .
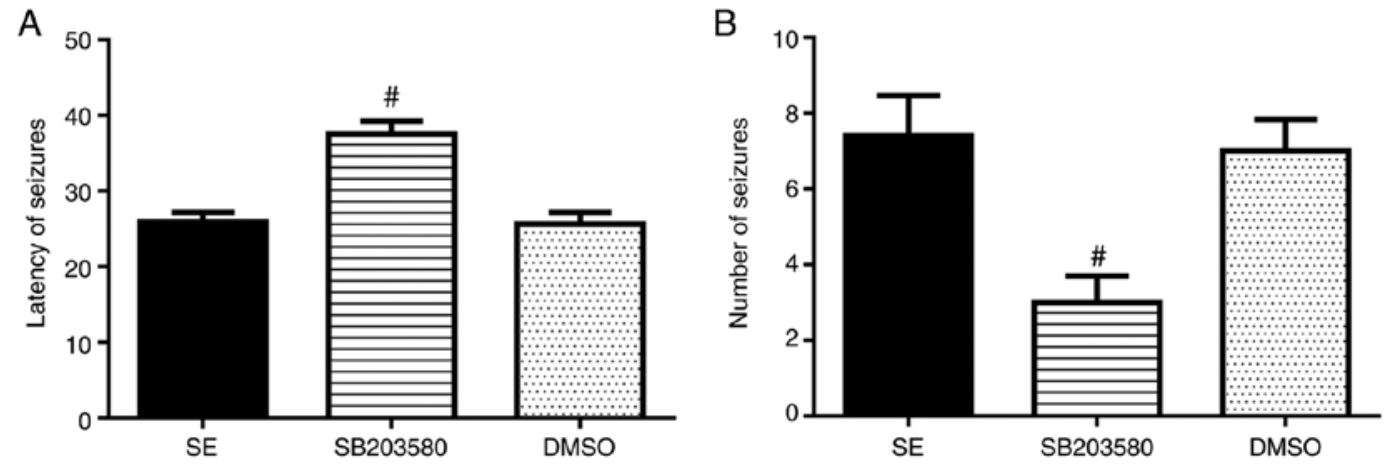

Figure 1. Effect of SB203580 on seizures. SB203580 (A) prolonged the latency of seizures and (B) decreased the number of seizures in experimental rats ( $\mathrm{n}=5$ /group). ${ }^{~} \mathrm{P}<0.05$ vs. SE. SE, status epilepticus.

with PBS with $1 \%$ Tween-20 (PBST) three times and then incubated with horseradish peroxidase (HRP)-conjugated secondary antibody (1:5,000; cat. no. 35105ES60; Shanghai Yeasen Biotechnology Co. Ltd) for $1 \mathrm{~h}$ at room temperature. Blots were developed using Super Signal West Pico Chemiluminescent HRP substrate (Sigma-Aldrich; Merck $\mathrm{KGaA}$ ) according to the manufacturer's instructions. Band intensity was analyzed with Quantity One software (Bio-Rad Laboratories, Inc.).

Immunofluorescence. Slides were incubated in acetone at $-20^{\circ} \mathrm{C}$ for $30 \mathrm{~min}$ washed in PBS three times for 5 min each, then $0.4 \%$ Triton $\mathrm{X}-100$ was added to cover the slices. The slices were placed in a $37^{\circ} \mathrm{C}$ incubator for $15 \mathrm{~min}$, washed with PBS three times for $5 \mathrm{~min}$ each, immersed in sodium citrate solution, heated at high heat $\left(92-98^{\circ} \mathrm{C}\right)$ in microwave oven for $3 \mathrm{~min}$ then at $37^{\circ} \mathrm{C}$ for $10 \mathrm{~min}$, and washed with PBS three times. The brain tissue slices were blocked in $20 \%$ goat serum (Beijing Solarbio Science \& Technology Co., Ltd.) for 1-3 h at $37^{\circ} \mathrm{C}$, after which the goat serum was discarded. The slides were incubated with rabbit anti-A1R (1:50; cat. no. ab82477; Abcam) and anti-ENT1 antibody (1:50; cat. no. ab223851; Abcam), to which a mixture of mouse anti-glial fibrillary acidic protein (GFAP; 1:100; cat. no. 3670S; Cell Signaling Technology, Inc.) and guinea pig anti-microtubule-associated protein 2 (MAP2) antibody (1:200; cat. no. 188004; Synaptic Systems $\mathrm{GmbH}$ ) was added. The sliced brain tissue was incubated at $4^{\circ} \mathrm{C}$ overnight. Then, the brain tissue slices were washed with PBS five times for $5 \mathrm{~min}$ each. The slides were incubated with a mixture of DyLight 488-conjugated goat anti-rabbit (1:50; cat. no. 4412S; Cell Signaling Technology, Inc), DyLight 594-conjugated goat anti-mouse (1:200; cat. no. ab96881; Abcam) and DyLight 650-conjugated goat anti-guinea pig antibody (1:50; cat. no. ab102377; Abcam) in a $37^{\circ} \mathrm{C}$ incubator in the dark for $1 \mathrm{~h}$. The slices were washed with PBS three times for $5 \mathrm{~min}$ each in the dark. Following the addition of $50 \%$ glycerol solution to cover the slide for storage, pictures were obtained with a laser confocal microscope (magnification, x200) and the slices were stored in the dark.

Immunohistochemistry. Paraffin sections (thickness, $4 \mu \mathrm{m}$ ) were deparaffinized, hydrated with an alcohol gradient and incubated in $0.3 \% \mathrm{H}_{2} \mathrm{O}_{2}$ for $15 \mathrm{~min}$ at room temperature. The sections were incubated for $5 \mathrm{~min}$ at $92-98^{\circ} \mathrm{C}$ in $10 \mathrm{mmol} / 1$ sodium citrate buffer and then blocked with $20 \%$ goat serum (Beijing Solarbio Science \& Technology Co., Ltd.) for $30 \mathrm{~min}$ at $37^{\circ} \mathrm{C}$. Then, the sections were incubated with rabbit anti-A1R antibody (1:100; cat. no. ab82477; Abcam) at $37^{\circ} \mathrm{C}$ for $2 \mathrm{~h}$ and washed four times with PBS. The sections were then incubated with biotinylated goat anti-rabbit secondary antibody (1:300; cat. no. TA130015; OriGene Technologies, Inc.) at room temperature for $30 \mathrm{~min}$, washed, treated with avidin-biotin complex solution for $30 \mathrm{~min}$ at $37^{\circ} \mathrm{C}$, washed with PBS and incubated with 3,3'-diaminobenzidine (DAB) (OriGene Technologies, Inc.) for $3 \mathrm{~min}$. Harris' hematoxylin was used for counterstaining at room temperature for $5 \mathrm{~min}$. Images were acquired with an automatic microscope (Nikon Corporation; magnification, x200).

Statistical analysis. Each experiment was repeated at least three times. Data are presented as the mean \pm SD and were analyzed using SPSS software (version 18.0; SPSS, Inc.). The behavioral scores of rats (latency of seizures and number of seizures) and results of immunohistochemistry and western 
A

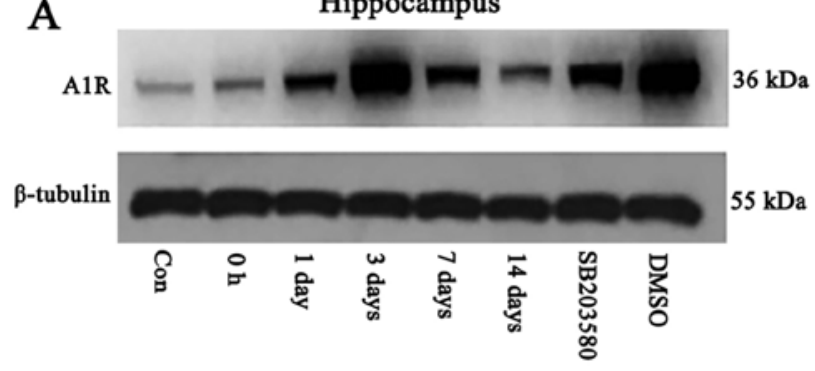

C

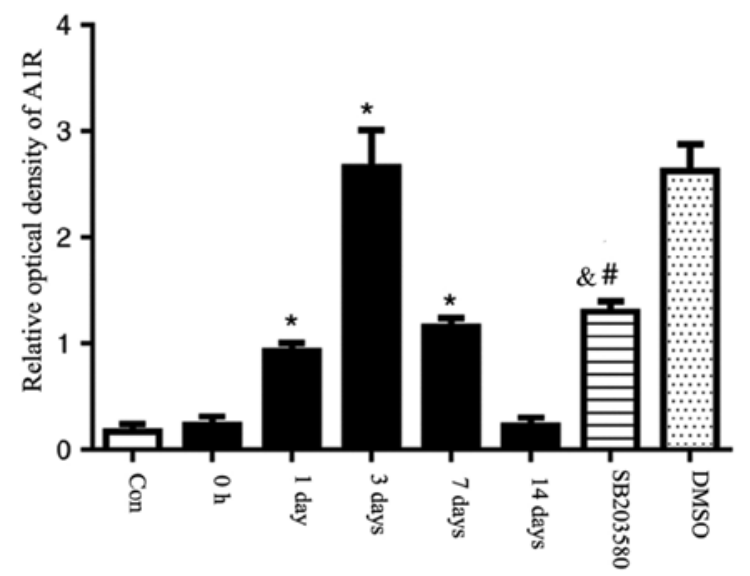

B
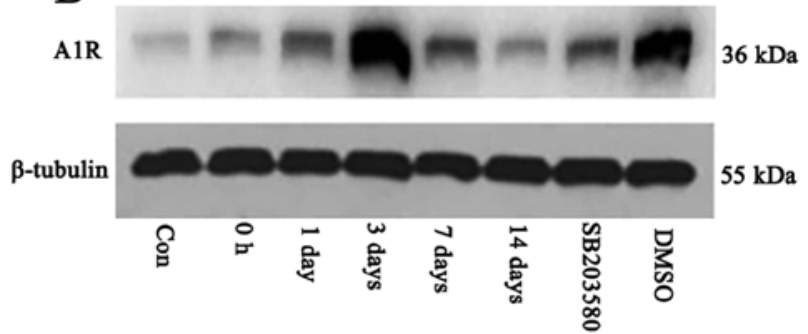

D

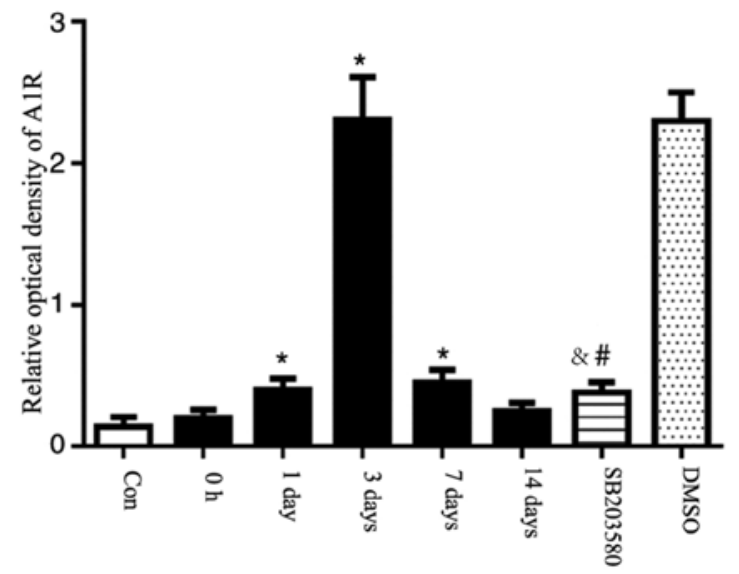

Figure 2. A1R protein was detected by western blot analysis. The relative optical density of A1R protein band was normalized to that of $\beta$-tubulin. A1R protein expression levels in the (A) hippocampus and (B) cortex of epileptic and con rats. Bar graphs for (C) hippocampus and (D) cortex of epileptic and con rats. Compared with con rats, A1R protein expression levels in the hippocampus of rats at 1 and 3 days and 7 days after seizure induction were upregulated. Compared with the con group, A1R protein expression levels were not significantly different at $0 \mathrm{~h}$ and 14 days. SB203580 inhibited A1R protein expression levels compared with epileptic rats at 3 days after seizure induction, whereas DMSO did not have this effect. $\mathrm{n}=5$ in each group. $\mathrm{P}<0.05$ vs. con; ${ }^{\#} \mathrm{P}<0.05$ vs. DMSO; ${ }^{\circledR} \mathrm{P}<0.05$ vs. 3 days. A1R, adenosine A1 receptor; con, control.

blot analysis were analyzed using one-way ANOVA followed by Tukey's multiple comparison test or repeated ANOVA. Student's t-test (unpaired) was used to analyze the immunofluorescence results between two groups. Normality and homogeneity of variance tests were performed prior to t-test and ANOVA. $\mathrm{P}<0.05$ was considered to indicate a statistically significant difference.

\section{Results}

SB203580 affects the latency of the first seizure and the number of epileptic seizures in rats. Number of seizures was significantly decreased and latency of seizures was prolonged in the SB203580 group compared with the SE group $(\mathrm{P}<0.05)$. No differences in these parameters between the DMSO and SE groups were observed ( $\mathrm{P}>0.05)$ (Fig. 1 and Table I).

AlR expression levels in the hippocampus and temporal lobe neocortex of epileptic rats. The expression levels of A1R in the hippocampus and temporal lobe neocortex of rats were detected by western blotting. The expression levels of A1R were significantly elevated at 1 and 3 days and 7 days after seizure induction. A1R expression levels peaked at 3 days, and were significantly different compared with the control group $(\mathrm{P}<0.05)$. No significant differences in the expression levels of $\mathrm{A} 1 \mathrm{R}$ at $0 \mathrm{~h}$ and 14 days after seizure induction were observed between the control and SE groups $(\mathrm{P}>0.05)$. An intervention

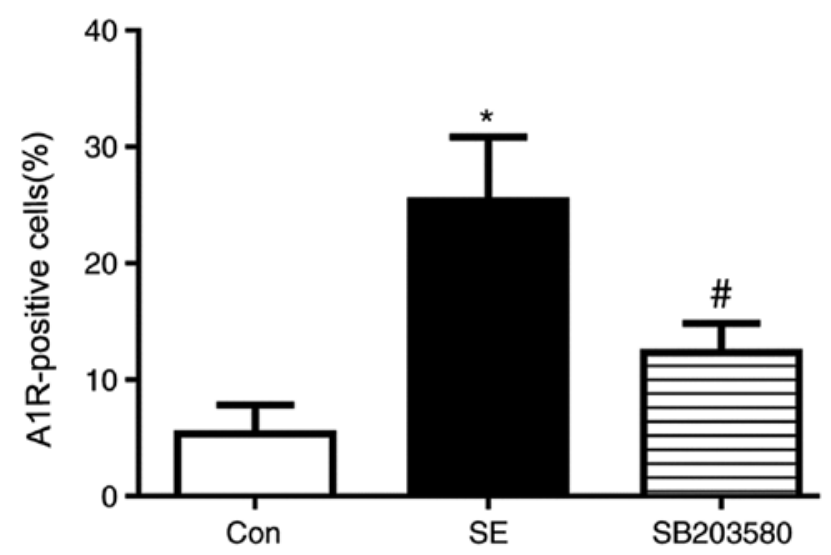

Figure 3. Statistical analysis of immunohistochemistry data showed that the percentage of A1R-positive cells was significantly increased in the SE group compared with the con group. Treatment with the p38 MAPK inhibitor SB203580 $(15 \mathrm{mg} / \mathrm{kg})$ decreased the expression levels of A1R compared with SE group. $\mathrm{n}=5$ in each group. ${ }^{*} \mathrm{P}<0.05$ vs. con; ${ }^{*} \mathrm{P}<0.05$ vs. $\mathrm{SE}$. A1R, adenosine A1 receptor; SE, status epilepticus; con, control.

time point of 3 days was chosen based on changes in A1R expression. Mice were pre-treated with SB203580, then, following 3 days of successful establishment of the epileptic seizure model, the brain tissue was taken for the next experiment, and DMSO was applied as the parallel solvent control. At day 3, A1R was expressed at significantly lower levels in 

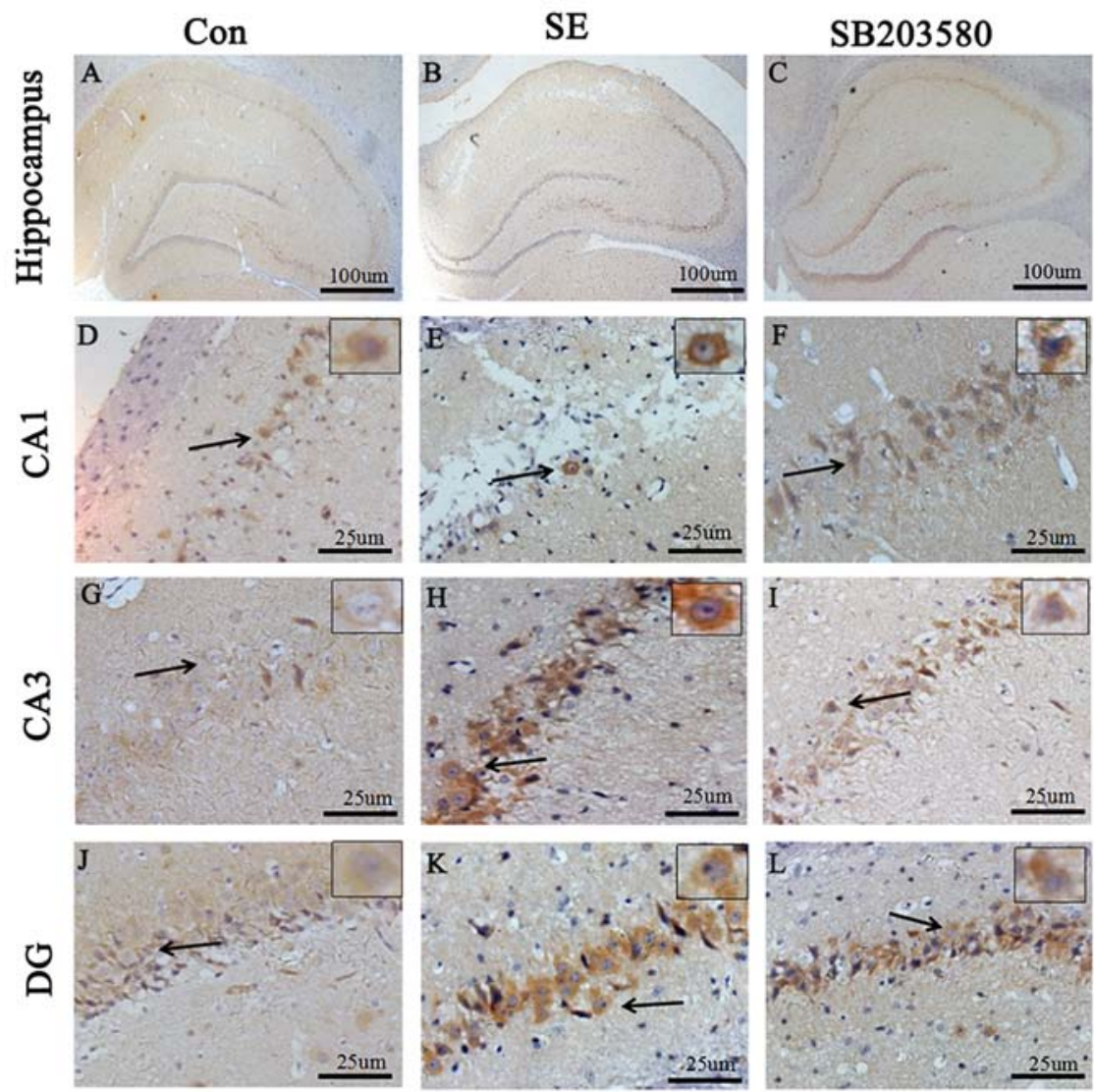

Figure 4. Immunohistochemical staining for A1R in the hippocampus of rats in the (A) Con, (B) SE (3 days) and (C) SB203580 groups at day 3. Scale bar, $100 \mu \mathrm{m}$. (D-F) CA1, (G-I) CA3 and (J-L) DG were divided into con group, SE group and SB203580 group from left to right. Scale bar, $25 \mu \mathrm{m}$. Black arrows indicate the A1R-positive neurons in the high-resolution image of the rectangular area. $\mathrm{n}=5 \mathrm{in}$ each group. A1R, adenosine A1 receptor; con, control; SE, status epilepticus; DG, dentate gyrus.

the SB203580 group than the SE groups $(\mathrm{P}<0.05)$, although the difference in A1R expression levels between the DMSO and the SE group was not statistically significant $(P>0.05$; Fig. 2). Preliminary experiments demonstrated that there was no difference in the expression levels of A1R in the normal, solvent and inhibitor groups at different time points (data not shown).

Immunohistochemical detection of $A 1 R$ expression levels in the hippocampus and temporal lobe neocortex. Compared with the control group, A1R protein levels in the hippocampus and neocortex of SE rats were elevated. Expression level profiles of A1R in the hippocampus and neocortex were assessed by immunohistochemistry. Positive staining for A1R was primarily detected in neurons located in the hippocampus (CA1 and CA3 regions and the dentate gyrus). In addition, Compared with the control group, the percentage of A1R-positive cells was significantly increased in the SE group. The difference in the percentage of A1R-positive cells between the SE and control groups was statistically significant $(\mathrm{P}<0.05)$. In addition, the percentage of A1R-positive cells was significantly decreased in the SB203580 group compared with the SE group, and the SB203580 group showed mild staining for A1R $(\mathrm{P}<0.05$; Figs. 3 and 4). There was no significant difference in the percentage of A1R-positive cells between the SE and DMSO groups ( $\mathrm{P}>0.05$; Figs. S1 and S2).
Localization of AlR in the hippocampus and temporal lobe neocortex. Immunohistochemical staining for A1R in the hippocampus and temporal cortex showed the expression levels of A1R in the hippocampal neurons of SE rats. The localization of A1R in the hippocampus and neocortex was compared between the control and the SE group (3 days after seizure induction) by multiple immunofluorescence staining. The fluorescence intensity of A1R in the hippocampus (CA1 and $\mathrm{CA} 3$ regions) and neocortex was significantly higher in the SE group compared with in the control group $(\mathrm{P}<0.05)$. A1R was expressed in cells labeled with dendrite-specific MAP2 but not in cells labeled with astrocyte-specific GFAP (Fig. 5).

Analysis of ENT1 expression levels in the hippocampus and temporal lobe neocortex of epileptic rats by western blotting. Western blotting was used to determine the basal expression levels of ENT1 protein in the hippocampus and temporal cortex of control rats. Compared with the control group, the protein expression levels of ENT1 in the hippocampus and temporal lobe neocortex were significantly increased in the SE group $(\mathrm{P}<0.05)$. However, compared with the SB203580 group, the protein expression levels of ENT1 in the hippocampus and temporal lobe neocortex were significantly increased in the $\mathrm{SE}$ group $(\mathrm{P}<0.05)$. No difference in ENT1 protein expression levels in the hippocampus and temporal lobe neocortex was observed between the DMSO and SE groups $(\mathrm{P}>0.05)$ (Figs. 6 and 7). 
A A1R

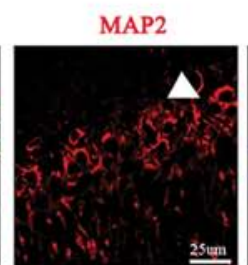

GFAP

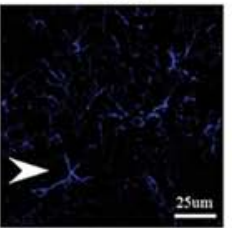

MERGE
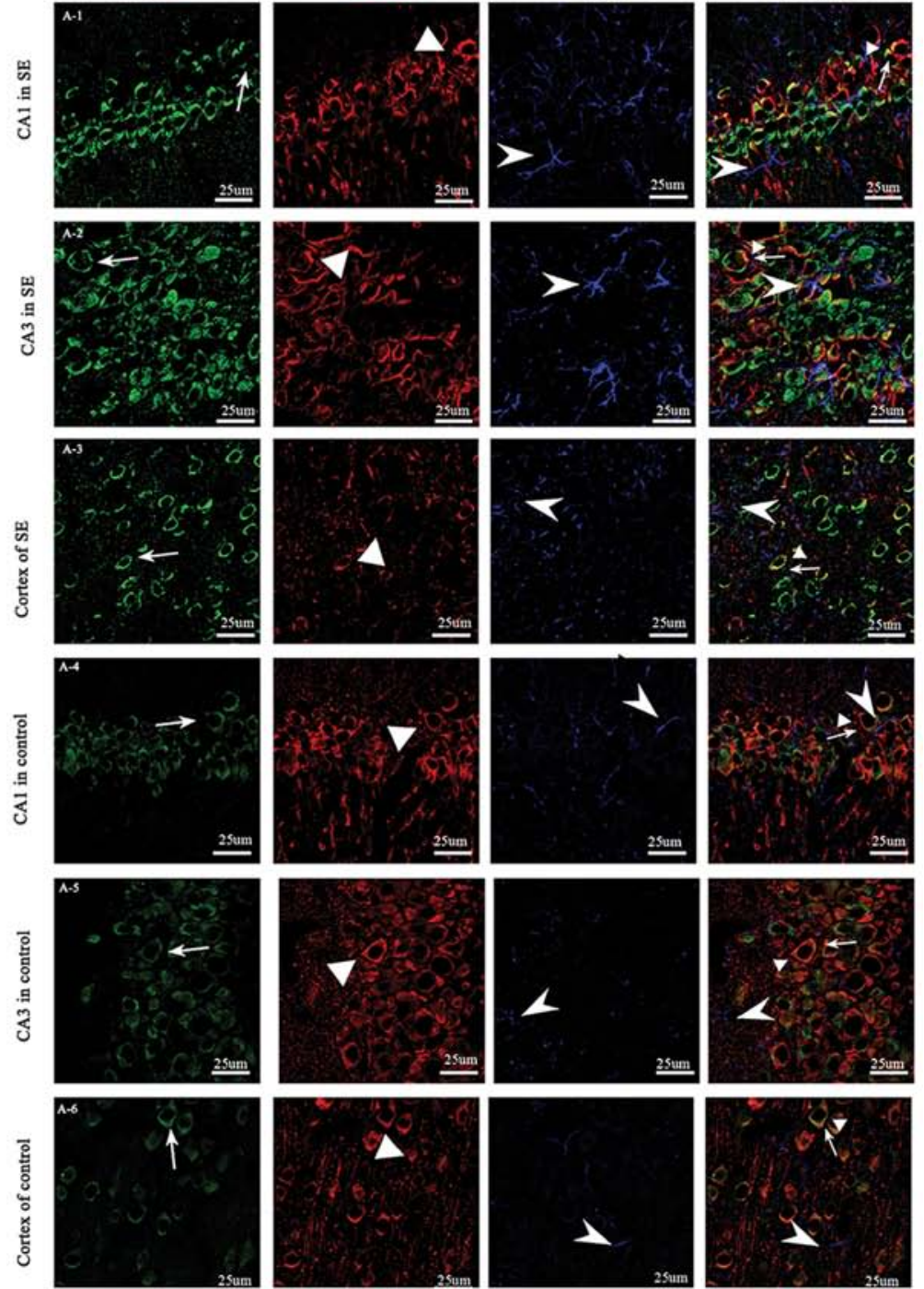

B

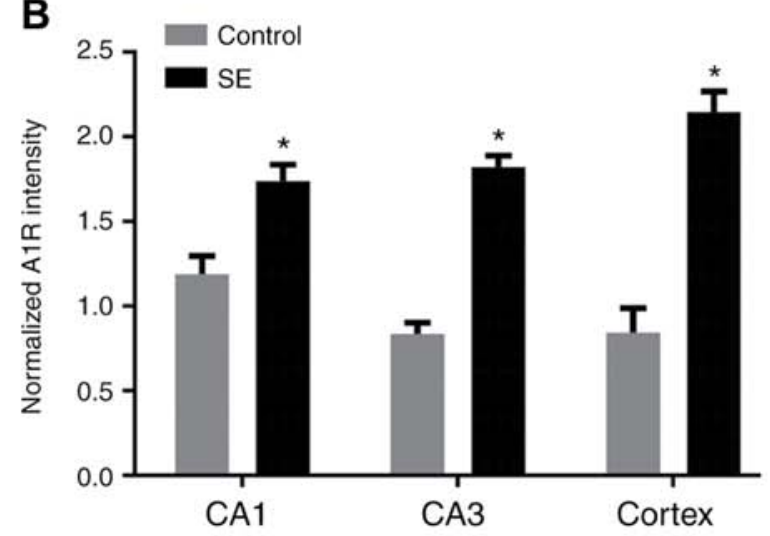

Figure 5. Localization of A1R in the hippocampus and temporal lobe neocortex. (A) Immunofluorescence staining showing A1R in the (A-1) CA1 and (A-2) CA3 regions of the hippocampus, and (A-3) temporal cortex in SE rats, and the (A-4) CA1 and (A-5) CA3 region, and (A-6) temporal cortex in control rats. A1R (green) was expressed in MAP2-positive neurons (red), but not GFAP-positive astrocytes (blue) in the temporal cortex and hippocampus (CA1 and $\mathrm{CA} 3$ regions). The fluorescence intensity of A1R was higher in the SE group than the control group, and A1R was expressed primarily in the neuronal cytoplasm. Line and arrow, A1R-positive cells; triangle, MAP2-positive cells; arrowhead, GFAP-positive cells. Scale bar, $25 \mu \mathrm{m}$. (B) Immunofluorescence analysis showed that the fluorescence intensity of A1R in the SE group was significantly higher than in the control group. "P<0.05 vs. control. A1R, adenosine A1 receptor; SE, status epilepticus; MAP, microtubule-associated protein; GFAP, glial fibrillary acidic protein.

Effect of the p38 MAPK inhibitor SB203580 on A1R expression levels. The western blotting results showed that A1R expression levels were upregulated in LiCl-pilocarpine SE model rats compared with control rats $(\mathrm{P}<0.05)$. By contrast, following administration of the p38 MAPK inhibitor SB203580, A1R expression levels in the SB203580 group were significantly 

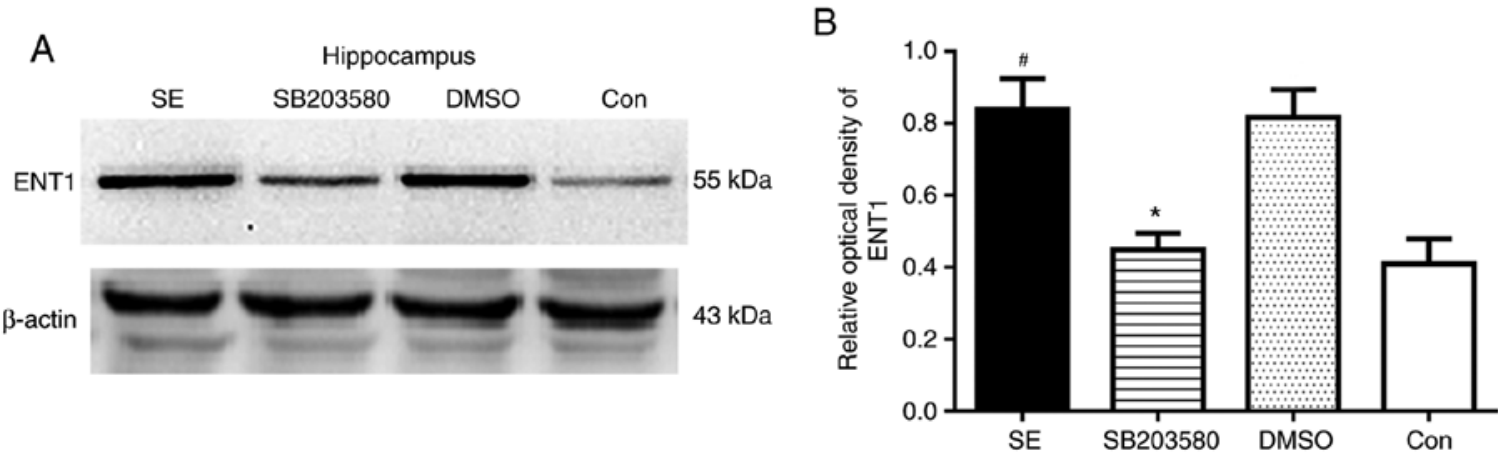

Figure 6. ENT1 protein expression levels in the hippocampus of epileptic and con rats. (A) ENT1 protein expression levels were detected by western blot analysis. The relative optical density values of ENT1 protein bands were normalized to those of $\beta$-actin. (B) Bar graphs demonstrating ENT1 expression levels in the hippocampus of epileptic and con rats. ENT1 protein expression was upregulated in the SE group 3 days after seizure induction. SB203580 inhibited ENT1 protein expression levels compared with that in SE rats 3 days after seizure induction, whereas DMSO did not have this effect. $\mathrm{n}=5$ in each group. ${ }^{*} \mathrm{P}<0.05$ vs. SE; ${ }^{\#} \mathrm{P}<0.05$ vs. con. ENT1, type 1 equilibrative nucleoside transporter; SE, status epilepticus; con, control.
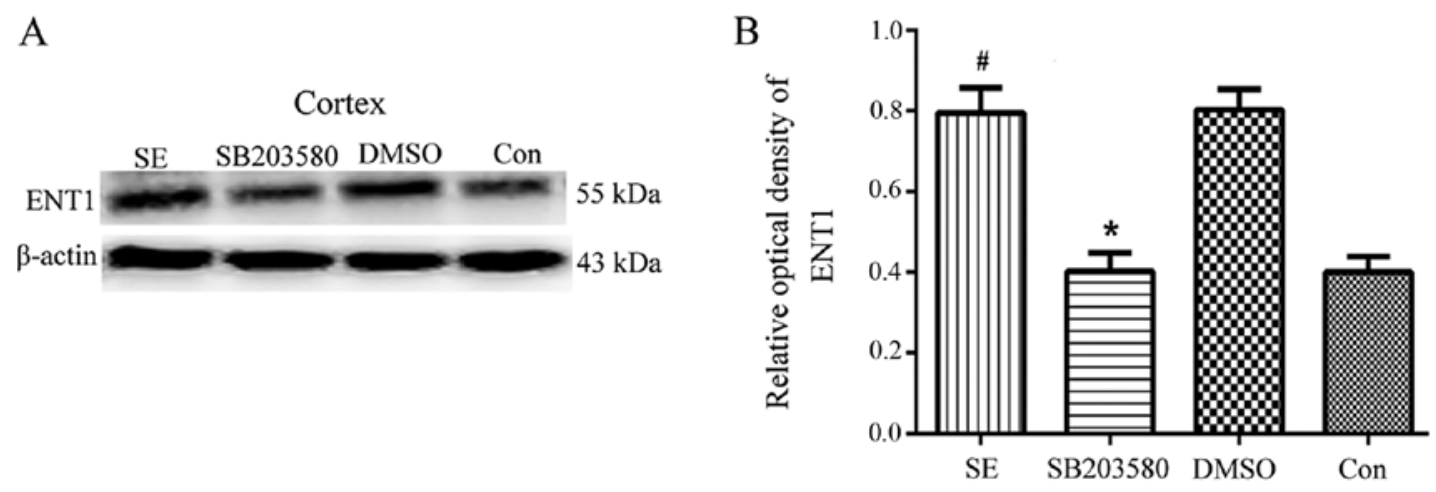

Figure 7. ENT1 protein expression levels in the cortex of epileptic and con rats. (A) ENT1 protein was detected by western blot analysis. The relative optical density of ENT1 protein bands was normalized to that of $\beta$-actin. (B) Respective bar graphs showing ENT1 protein expression levels in the cortex of epileptic and con rats. ENT1 protein expression levels were upregulated in the SE group 3 days after seizure induction. SB203580 inhibited ENT1 protein expression levels compared with SE rats 3 days after seizure induction, whereas DMSO did not have this effect. $\mathrm{n}=5$ in each group. " $\mathrm{P}<0.05$ vs. SE; ${ }^{*} \mathrm{P}<0.05$ vs. con. ENT1, type 1 equilibrative nucleoside transporter; con, control; SE, status epilepticus.
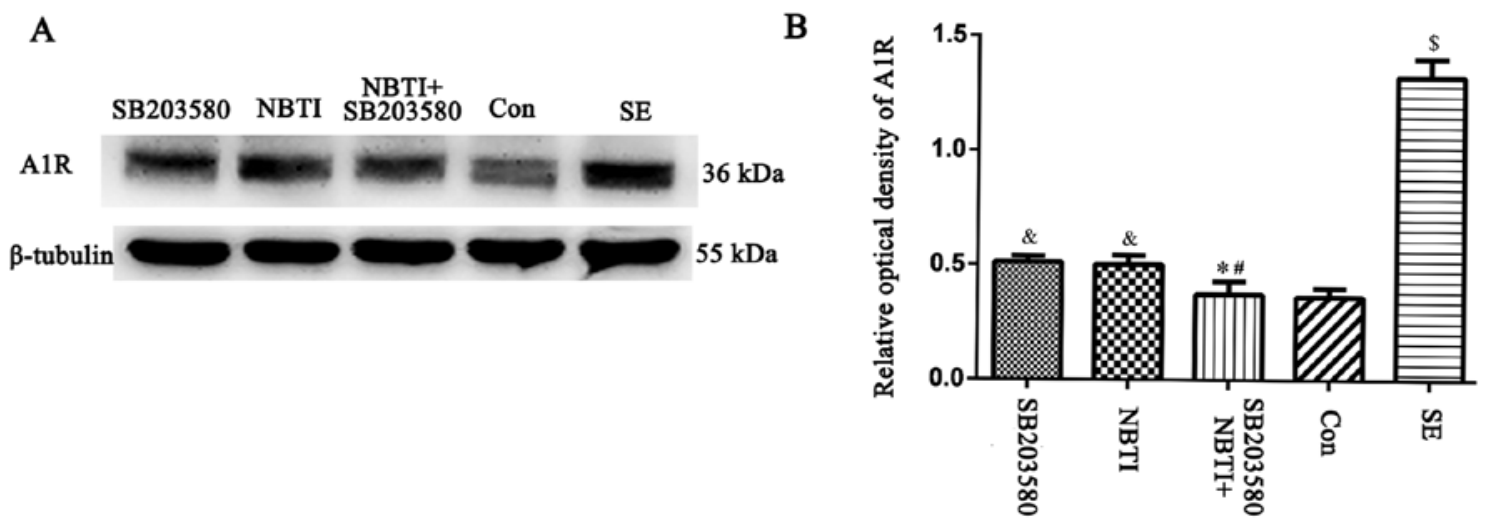

Figure 8. Expression levels of the A1R protein in the hippocampus of rats in the NBTI (type 1 equilibrative nucleoside transporter inhibitor), SB203580 (p38 MAPK inhibitor), SE, NBTI + SB203580 and con groups. (A) A1R protein was detected by western blotting. The relative optical density of the A1R protein band was normalized to that of $\beta$-tubulin. (B) Bar graphs showing A1R expression levels in the hippocampus of rats from each group. Compared with the con group, expression levels of A1R in the SE group were upregulated. A1R levels in the SB203580 were downregulated compared with the SE group. The expression levels of A1R was lower in the NBTI group than the SE group. There was no significant difference between the expression levels of A1R in the NBTI and SB203580 groups. Compared with the NBTI and SB203580 groups, the expression levels of A1 receptor were downregulated in the NBTI + SB203580 group. $\mathrm{n}=5$ in each group. ${ }^{\mathrm{P}}<0.05$ vs. SB203580; ${ }^{\mathrm{P}}<0.05$ vs. NBTI; ${ }^{\circledR} \mathrm{P}<0.05$ vs. SE; ${ }^{\$} \mathrm{P}<0.05$ vs. con. A1R, adenosine A1 receptor; NBTI, nitrobenzothioinosine; con, control; SE, status epilepticus.

lower than in the SE group at 3 days after seizure induction $(\mathrm{P}<0.05)$. Following administration of the ENT1 inhibitor
NBTI, the expression levels of A1R in the NBTI group were significantly lower than in the SE group at 3 days after seizure 


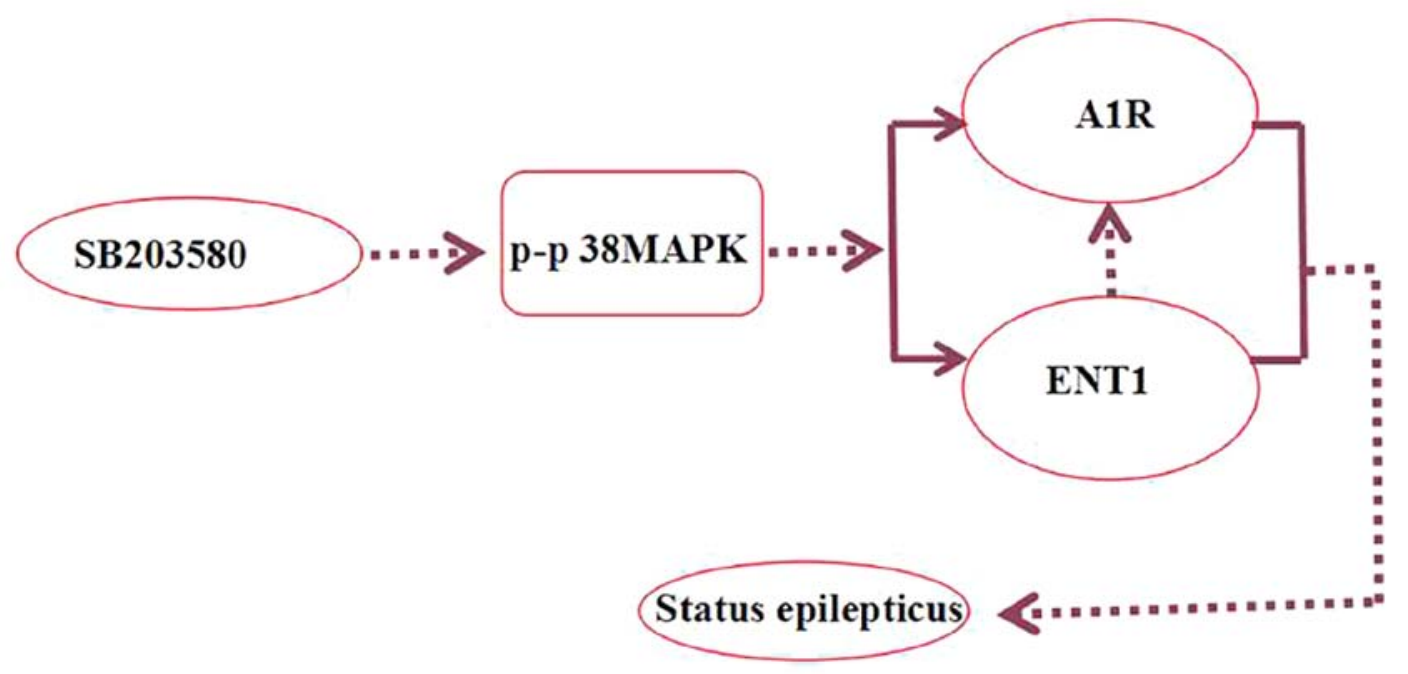

Figure 9. SB203580 inhibits the activation of p-p38 MAPK, regulates A1R and ENT1, and decreases the number of seizures and brain tissue damage. Dashed arrows represent suppression. p-, phosphorylated; A1R, adenosine A1 receptor; ENT1, type 1 equilibrative nucleoside transporter.

induction $(\mathrm{P}<0.05)$. By contrast, no significant difference in A1R expression levels was observed between the NBTI and SB203580 groups ( $\mathrm{P}>0.05)$. In addition, following administration of NBTI + SB203580, A1R expression levels were downregulated at 3 days compared with NTI alone. In addition, a significant decrease in A1R expression levels was observed between the NBTI and SB203580 groups ( $\mathrm{P}<0.05$; Fig. 8).

\section{Discussion}

$\mathrm{SE}$ is defined as the occurrence of sustained seizures for $>5$ min (30). Due to the need for timely termination of SE, delayed intervention of seizures, particularly tonic-clonic seizures, may lead to nervous system injury and death (31). $\mathrm{SE}$ is a common, acute and severe neurological disease with high mortality that is characterized by abnormal discharge of various stimuli by highly synchronized neurons in the brain, including neuroinflammation and oxidative stress (32). Seizures are estimated to account for $0.75 \%$ of the global disease burden, based on the research in predominantly lowand middle-income countries (33). SE needs to be terminated in a timely manner; the most common and important method is drug therapy $(34,35)$. Currently used antiepileptic drugs primarily inhibit nerve excitation, thereby inhibiting epileptic seizure (36). However, in $~ 30 \%$ of individuals with epilepsy, existing antiepileptic drugs do not control repeated epileptic seizures (36). Therefore, studies aiming to identify novel treatments for patients with epilepsy remain important (37-39).

Adenosine is present in all cells and is involved in controlling the function of all types of tissue and organ. Elevated adenosine levels prevent cell damage and organ dysfunction (40). In addition, adenosine is involved in the adjustment of pathophysiological processes, such as the management of sleep and wakefulness, and affects postsynaptic receptors involved in the release of neurotransmitters (such as glutamate, acetylcholine, thyroxine, serotonin and dopamine) (40). The adenosine system exerts inhibitory effects on the brain; therefore, adenosine is regarded as an endogenous anticonvulsant (15). The anticonvulsant effect of adenosine is primarily mediated by $\mathrm{A} 1 \mathrm{R}$ and adenosine $\mathrm{A} 2 \mathrm{~A}$ receptors, which are in dynamic equilibrium in normal brain tissue and maintain the inhibitory effect of the adenosine system (41). A1R inhibits voltage-gated $\mathrm{Ca} 2{ }^{+}$channels, preventing glutamate release and neuronal depolarization (42). In addition, A1R activates $\mathrm{K}^{+}$channels to enhance potassium ion outflow, resulting in the hyperpolarization of postsynaptic membrane and reducing the excitability of nerve conduction (43). The adenosine A2A receptor is primarily expressed in the thalamus, where it functions to promote excitatory synaptic transmission and offset the inhibitory effect of A1R upon glutamate release (44). Thus, the adenosine system is regarded as an 'endogenous antiepilepsy system' (45).

In the present study, dynamic changes in A1R expression levels following SE were observed in rats. A1R expression levels began to increase 1 day after successful establishment of the SE model and peaked after 3 days before gradually decreasing over 1-2 weeks after seizure induction. Based on the immunohistochemistry results, the number of A1R-positive cells increased and staining for A1R was enhanced in the SE group at 3 days after seizure induction compared with the control group. In the normal brain, A1R and adenosine receptor A2A exhibit a dynamic equilibrium, maintaining the inhibitory effects of the adenosine system. In previous studies in which changes in the expression levels of adenosine receptors in a rat model of the acute phase of epilepsy were examined, A1R was significantly increased 1 day after seizure induction, thus indicating an adaptive response to an acute attack $(34,38)$. However, in patients and animal models of chronic epilepsy, A1R expression levels in brain tissue are downregulated, whereas A2A receptor expression levels are notably upregulated, leading to inhibition of A1R (46). This decrease in A1R expression levels is considered to reflect nerve cell degeneration, and increased extracellular adenosine and A1R expression levels have been revealed to improve the inhibitory effects of the adenosine system until the end of a seizure (15).

In the present study, the seizure onset latency was prolonged and the number of seizures decreased in rats with acute epilepsy treated with the p38 MAPK inhibitor SB203580. SB203580 has previously been demonstrated to selectively inhibit the 
activation of p38 MAPK, decrease epileptic seizures in rats and protect CA3 neurons, indicating that the p38 MAPK signaling pathway is involved in an antiepileptic mechanism in the brain (47). In SE, the absence of neurons also increases the expression levels of phosphorylated-p38 and inhibition of p38 MAPK decreases neuronal damage (48). Studies have revealed that the adenosine system activates multiple MAPK signaling pathways via G-protein coupling $(49,50)$. ENT1 is a subtype of a nucleoside transporter and its primary function is to regulate the level of adenosine (51). In the present study, the p38 MAPK inhibitor SB203580 decreased the number of seizures and brain tissue damage, as well as stress-induced endogenous A1R expression levels (Fig. 9).

Numerous types of nervous system disease (including epilepsy, neurodegenerative disease, mental disorders and cerebral blood disease) may involve nucleosides; nucleoside drugs have been designed to treat a range of types of tumors and viral infections $(52,53)$. However, the intra- and extracellular availability of nucleosides and their derivatives depends on the presence of specific nucleoside transporters (54). Importantly, our previous study found that i.p. and hippocampal injections of NBTI, a selective ENT1 inhibitor, decreased the number of seizures and prolonged the latency of the first seizure in rats, indicating that ENT1 plays an important role in the development of epilepsy (29). Therefore, ENT1 may be a novel therapeutic target for the control of epileptic seizures in the clinic $(22,28)$. Based on the results of western blot analysis in the present study, ENT1 expression levels 3 days after seizure induction were significantly lower in the SB203580 group than in the epilepsy group, showing that inhibition of the p38 MAPK signaling pathway modified ENT1 expression levels in epileptic rats.

There were certain limitations of the present study. Following SE, the distribution of A1R inside and outside of cells was different from that of normal rats; increased extracellular A1R can protect neurons (55). Secondly, SB203580 was used to inhibit p38 MAPK, but changes in p38 levels following SB203580 application were not detected. Immunohistochemistry demonstrated that the SB203580 group had fewer A1R-positive cells than the SE group; future immunofluorescence experiments should compare the fluorescence intensity of the SE and normal control groups. Moreover, changes in fluorescence intensity of A1R in the SB203580 group require further investigation.

In summary, the results of the present study demonstrated that the p38 MAPK inhibitor SB203580 decreased pathological damage to hippocampal neurons, prolonged the seizure latency and decreased A1R and ENT1 protein expression levels in rats. The proposed antiepileptic effect and protective effects of SB203580 in neurons may be due to decreased ENT1 expression levels, although the specific mechanism requires further investigation.

\section{Acknowledgements}

Not applicable.

\section{Funding}

The present study was supported by grants from the National Natural Science Foundation of China (grant nos. 81560224 and 81660227) and the Guizhou Provincial Science and Technology Foundation [grant nos. LH (2015) 7520 and LH (2015) 7471].

\section{Availability of data and materials}

The datasets used and/or analyzed during the current study are available from the corresponding author on reasonable request.

\section{Authors' contributions}

ZX, XZ and QC conceived and designed the study. XZ, QC, $\mathrm{YC}, \mathrm{HZ}$ and $\mathrm{HH}$ performed the experiments. JZh, JW, YP, JZe and $\mathrm{ZF}$ conducted statistical analysis, $\mathrm{XZ}$ and $\mathrm{QC}$ wrote the paper. XZ, QC, HH, JZh, JW, YP, JZe and ZF reviewed and edited the manuscript. All authors read and approved the final manuscript.

\section{Ethics approval and consent to participate}

All procedures were performed in accordance with the Guide for and Use of Medical Laboratory Animals (Ministry of Health of China, 1998) and approved by Animal experiment ethics committee of Zunyi Medical College [approval no. (2016) 2-044].

\section{Patient consent for publication}

Not applicable.

\section{Competing interests}

The authors declare that they have no competing interests.

\section{References}

1. Mallok A, Vaillant JD, Soto MT, Viebahn-Hänsler R, Viart ML, Pérez AF, Cedeño RI and Fernández OS: Ozone protective effects against PTZ-induced generalized seizures are mediated by reestablishment of cellular redox balance and A1 adenosine receptors. Neurol Res 37: 204-210, 2015.

2. Lawson T and Yeager S: Status epilepticus in adults: A review of diagnosis and treatment. Crit Care Nurse 36: 62-73, 2016.

3. Sutter R, Semmlack S and Kaplan PW: Nonconvulsive status epilepticus in adults - insights into the invisible. Nat Rev Neurol 12: 281-293, 2016.

4. Córdova-Dávalos L, Carrera-Calvo D, Solís-Navarrete J, Mercado-Gómez OF, Arriaga-Ávila V, Agredano-Moreno LT, Jiménez-García LF and Guevara-Guzmán R: Status epilepticus triggers early mitochondrial fusion in the rat hippocampus in a lithium-pilocarpine model. Epilepsy Res 123: 11-19, 2016.

5. Cui Y, Liu J, Luo Y, He S, Xia Y, Zhang Y, Yao D and Guo D: Aberrant connectivity during pilocarpine-induced status epilepticus. Int J Neural Syst 30: 1950029, 2020.

6. Xie L, Li T, Song X, Sun H, Liu J, Yang J, Zhao W, Cheng L, Chen H, Liu B, et al: Dynamic alteration of dendrites and dendritic spines in the hippocampus and microglia in mouse brain tissues after kainate-induced status epilepticus. Int J Neurosci: Jun 1, 2020 (Epub ahead of print).

7. $\mathrm{Hu} \mathrm{JH}$, Malloy C and Hoffman DA: P38 Regulates kainic acid-induced seizure and neuronal firing via Kv4.2 phosphorylation. Int J Mol Sci 21: E5921, 2020.

8. Che Y, Yu YM, Han PL and Lee JK: Delayed induction of p38 MAPKs in reactive astrocytes in the brain of mice after KA-induced seizure. Brain Res Mol Brain Res 94: 157-165, 2001.

9. Neligan A and Shorvon SD: Frequency and prognosis of convulsive status epilepticus of different causes: A systematic review. Arch Neurol 67: 931-940, 2010. 
10. Matute $\mathrm{C}$ and Cavaliere F: Neuroglial interactions mediated by purinergic signalling in the pathophysiology of CNS disorder. Semin Cell Dev Biol 22: 252-259, 2011.

11. Li T, Quan Lan J, Fredholm BB, Simon RP and Boison D Adenosine dysfunction in astrogliosis: Cause for seizure generation? Neuron Glia Biol 3: 353-366, 2007.

12. Masino SA, Kawamura M Jr and Ruskin DN: Adenosine receptors and epilepsy: Current evidence and future potential. Int Rev Neurobiol 119: 233-255, 2014

13. Boison D: Adenosine dysfunction in epilepsy. Glia 60: 1234-1243, 2012.

14. Cunha RA. How does adenosine control neuronal dysfunction and neurodegeneration. J Neurochem. 2016.139(6): 1019-1055.

15. Hargus NJ, Jennings C, Perez-Reyes E, Bertram EH and Patel MK: Enhanced actions of adenosine in medial entorhinal cortex layer II stellate neurons in temporal lobe epilepsy are mediated via A(1)-receptor activation. Epilepsia 53: 168-176, 2012.

16. Ono $\mathrm{K}$ and Han J: The p38 signal transduction pathway: Activation and function. Cell Signal 12: 1-13, 2000.

17. Downey JS and Han J: Cellular activation mechanisms in septic shock. Front Biosci 3: d468-d476, 1998.

18. Robinson MJ and Cobb MH: Mitogen-activated protein kinase pathways. Curr Opin Cell Biol 9: 180-186, 1997.

19. Okamoto OK, Janjoppi L, Bonone FM, Pansani AP, da Silva AV Scorza FA and Cavalheiro EA: Whole transcriptome analysis of the hippocampus: Toward a molecular portrait of epileptogenesis. BMC Genomics 11: 230, 2010.

20. Yang Z, Wang J, Yu C, Xu P, Zhang J, Peng Y, Luo Z, Huang H Zeng $\mathrm{J}$ and $\mathrm{Xu} Z$ : Inhibition of $\mathrm{p} 38 \mathrm{MAPK}$ signaling regulates the expression of EAAT2 in the brains of epileptic rats. Front Neurol 9: 925, 2018

21. Minor TR, Rowe M, Cullen PK and Furst S: Enhancing brain adenosine signaling with the nucleoside transport blocker NBTI (S-(4-nitrobenzyl)-6-theoinosine) mimics the effects of inescapable shock on later shuttle-escape performance in rats. Behav Neurosci 122: 1236-1247, 2008.

22. Huang H, Wang J, Zhang J, Luo Z, Li D, Qiu X, Peng Y, Xu Z, $\mathrm{Xu} \mathrm{P}$ and $\mathrm{Xu} \mathrm{Z}$ : Nitrobenzylthioinosine mimics adenosine to attenuate the epileptiform discharge of hippocampal neurons from epileptic rats. Oncotarget 8: 35573-35582, 2017.

23. Boison D: Adenosine as a modulator of brain activity. Drug News Perspect 20: 607-611, 2007.

24. Huang M, Wang Y, Collins M, Gu JJ, Mitchell BS and Graves LM Inhibition of nucleoside transport by p38 MAPK inhibitors. J Biol Chem 277: 28364-28367, 2002.

25. Rashid K, Van der Zee CE, Ross GM, Chapman CA, Stanisz J Riopelle RJ, Racine RJ and Fahnestock M: A nerve growth factor peptide retards seizure development and inhibits neuronal sprouting in a rat model of epilepsy. Proc Natl Acad Sci USA 92: 9495-9499, 1995.

26. Bulavin DV, Phillips C,Nannenga B, Timofeev O, Donehower LA, Anderson CW, Appella E and Fornace AJ Jr: Inactivation of the Wip1 phosphatase inhibits mammary tumorigenesis through $\mathrm{p} 38$ MAPK-mediated activation of the p16(Ink4a)-p19(Arf) pathway. Nat Genet 36: 343-350, 2004

27. Li Q, Liu H, Du J, Chen B, Li Q, Guo X, Huang X and Huang Q: Advanced glycation end products induce moesin phosphorylation in murine brain endothelium. Brain Res 1373: 1-10, 2011.

28. Mannangatti P, NarasimhaNaidu K, Damaj MI, Ramamoorthy S and Jayanthi LD: A Role for p38 Mitogen-activated Protein Kinase-mediated Threonine 30-dependent Norepinephrine Transporter Regulation in Cocaine Sensitization and Conditioned Place Preference. J Biol Chem 290: 10814-10827, 2015.

29. Xu Z, Xu P, Chen Y, Liu J, Zhang Y, Lv Y, Luo J, Fang M, Zhang J, Wang J, et al: ENT1 inhibition attenuates epileptic seizure severity via regulation of glutamatergic neurotransmission. Neuromolecular Med 17: 1-11, 2015.

30. Marawar R, Basha M, Mahulikar A, Desai A, Suchdev K and Shah A: Updates in refractory status epilepticus. Crit Care Re Pract 2018: 9768949, 2018.

31. Smith PE: Management of Established Status Epilepticus. N Engl J Med 381: 2171-2172, 2019.

32. Terrone G, Frigerio F, Balosso S, Ravizza T and Vezzani A Inflammation and reactive oxygen species in status epilepticus: Biomarkers and implications for therapy. Epilepsy Behav 101 (Pt B): 106275, 2019.

33. Thijs RD, Surges R, O'Brien TJ and Sander JW. Epilepsy in adults. Lancet 393: 689-701, 2019.
34. Goodrich GS, Kabakov AY, Hameed MQ, Dhamne SC, Rosenberg PA and Rotenberg A: Ceftriaxone treatment after traumatic brain injury restores expression of the glutamate transporter, GLT-1, reduces regional gliosis, and reduces post-traumatic seizures in the rat. J Neurotrauma 30: 1434-1441, 2013.

35. Wagner RG, Bottomley C, Ngugi AK, Ibinda F, Gómez-Olivé FX, Kahn K, Tollman S, Newton CR, Wagner R, Twine R, et al; SEEDS Writing Group: Incidence, remission and mortality of convulsive epilepsy in rural northeast South Africa. PLoS One 10: e0129097, 2015.

36. Arnold S. Cenobamate: new hope for treatment-resistant epilepsy. Lancet Neurol 19: 23-24, 2020.

37. Boison D: The Biochemistry and Epigenetics of Epilepsy: Focus on Adenosine and Glycine. Front Mol Neurosci 9: 26, 2016.

38. Kwan P and Brodie MJ: Early identification of refractory epilepsy. N Engl J Med 342: 314-319, 2000.

39. Shao Y, Wang C, Hong Z and Chen Y: Inhibition of p38 mitogen-activated protein kinase signaling reduces multidrug transporter activity and anti-epileptic drug resistance in refractory epileptic rats. J Neurochem 136: 1096-1105, 2016.

40. Świąder MJ, Kotowski J and Łuszczki JJ: Modulation of adenosinergic system and its application for the treatment of epilepsy. Pharmacol Rep 66: 335-342, 2014.

41. Sebastião AM and Ribeiro JA: Adenosine receptors and the central nervous system. Handb Exp Pharmacol 193: 471-534, 2009.

42. Fredholm BB, Chen JF, Cunha RA, Svenningsson P and Vaugeois JM: Adenosine and brain function. Int Rev Neurobiol 63: 191-270, 2005

43. Liu YJ, Chen J, Li X, Zhou X, Hu YM, Chu SF, Peng Y and Chen NH: Research progress on adenosine in central nervous system diseases. CNS Neurosci Ther 25: 899-910, 2019.

44. Boison D, Chen JF and Fredholm BB: Adenosine signaling and function in glial cells. Cell Death Differ 17: 1071-1082, 2020.

45. Boison D: Adenosine as a modulator of brain activity. Drug News Perspect 20: 607-611, 2007.

46. Huicong K, Zheng X, Furong W, Zhouping T, Feng X, Qi H, Xiaoyan L, Xiaojiang $\mathrm{H}, \mathrm{Na} Z$, Ke X, et al: The imbalanced expression of adenosine receptors in an epilepsy model corrected using targeted mesenchymal stem cell transplantation. Mol Neurobiol 48: 921-930, 2013

47. Jiang W, Van Cleemput J, Sheerin AH, Ji SP, Zhang Y, Saucier DM, Corcoran ME and Zhang X: Involvement of extracellular regulated kinase and p38 kinase in hippocampal seizure tolerance. J Neurosci Res 81: 581-588, 2005.

48. Cieślak M, Wojtczak A and Komoszyński M: Role of the purinergic signaling in epilepsy. Pharmacol Rep 69: 130-138, 2017.

49. Kim DS, Min SJ, Kim MJ, Kim JE and Kang TC: Leptomycin B ameliorates vasogenic edema formation induced by status epilepticus via inhibiting p38 MAPK/VEGF pathway. Brain Res 1651: 27-35, 2016.

50. Akhtar S, Yousif MH, Chandrasekhar B and Benter IF: Activation of EGFR/ERBB2 via pathways involving ERK1/2, P38 MAPK, $\mathrm{AKT}$ and FOXO enhances recovery of diabetic hearts from ischemia-reperfusion injury. PLoS One 7: e39066, 2012.

51. Zhang YN, Dong HT, Yang FB, Wang ZQ, Ma ZH, Ma SZ, Ma XD and Duan L: Nrf2-ARE signaling pathway regulates the expressions of A1R and ENT1 in the brain of epileptic rats. Eur Rev Med Pharmacol Sci 22: 6896-6904, 2018.

52. Boison D: Adenosine as a neuromodulator in neurological diseases. Curr Opin Pharmacol 8: 2-7, 2008.

53. Zhang J, Visser F, King KM, Baldwin SA, Young JD and Cass CE: The role of nucleoside transporters in cancer chemotherapy with nucleoside drugs. Cancer Metastasis Rev 26: 85-110, 2007.

54. Parkinson FE, Damaraju VL, Graham K, Yao SY, Baldwin SA, Cass CE and Young JD: Molecular biology of nucleoside transporters and their distributions and functions in the brain. Curr Top Med Chem 11: 948-972, 2011.

55. Rombo DM, Dias RB, Duarte ST, Ribeiro JA, Lamsa KP and Sebastião AM: Adenosine A1 receptor suppresses tonic GABAA receptor currents in hippocampal pyramidal cells and in a defined subpopulation of interneurons. Cereb Cortex 26: 1081-1095, 2016.

This work is licensed under a Creative Commons Attribution-NonCommercial-NoDerivatives 4.0 International (CC BY-NC-ND 4.0) License. 